Journal of Advanced Research in Fluid Mechanics and Thermal Sciences

\title{
Gravity-Aided Ultrasonic Separation of Nanoparticles from Liquid Using Macro-Scale Separator
}

\author{
Khin Nwe Zin Tun ${ }^{1,}$, Khine Zin Mar ${ }^{1}$, Thein Min Htike ${ }^{1}$ \\ 1 Department of Mechanical Engineering, Yangon Technological University, Myanmar
}

\begin{tabular}{|c|c|}
\hline ARTICLE INFO & ABSTRACT \\
\hline $\begin{array}{l}\text { Article history: } \\
\text { Received } 2 \text { October } 2019 \\
\text { Received in revised form } 16 \text { December } 2020 \\
\text { Accepted } 16 \text { December } 2020 \\
\text { Available online } 15 \text { January } 2021\end{array}$ & $\begin{array}{l}\text { Acoustophoresis is the technology to separate microparticles and cells from } \\
\text { suspending fluid. This research focuses on the separation of nanoparticles from water } \\
\text { by using macro-scale fluidic separator which works based on gravity-aided ultrasonic } \\
\text { standing wave technology. Titanium dioxide particles of diameter ( } 40 \mathrm{~nm} \text { to } 100 \mathrm{~nm} \text { ) } \\
\text { were concentrated by the combination of ultrasonic standing wave field at } 2.2 \mathrm{MHz} \\
\text { and gravity-aided sedimentation. The purpose of this study is to investigate the } \\
\text { performance of gravity-aided ultrasonic particle separator to concentrate } \\
\text { nanoparticles. It was found that the separation efficiency is } 82.85 \% \text { at a flow rate of } \\
0.1 \mathrm{~mL} / \mathrm{min} \text {. FEM simulations were also conducted to evaluate characteristics of } \\
\text { variation of acoustic energy inside the fluidic channel. Results indicate that } \\
\text { nanoparticles can be concentrated using gravity-aided ultrasonic standing wave field, } \\
\text { however optimization of the design of the fluidic channel is required for increasing } \\
\text { throughput of the separator. }\end{array}$ \\
\hline
\end{tabular}

Keywords:

ultrasonic standing wave; acoustic radiation force; macro-scale fluidic separator; microparticles

Copyright @ 2021 PENERBIT AKADEMIA BARU - All rights reserved

\section{Introduction}

The Particle separation methods have wide applications in engineering and life sciences. Ultrasonic standing wave is one technology used in manipulating particles in microfluidic systems. This method is suitable for both biological and non-biological micro-particles suspended in a fluid medium. The particle separation using ultrasonic standing wave relies on acoustic radiation force being directed on the particles either in a macro- or micro-channel both with flowing and stationary fluid volumes. The acoustic radiation force depends on not only the intensity of the sound field but also the contrast between the mechanical parameters density and compressibility of the particles and the fluid.

Millimetre-scale h-shaped ultrasonic concentrator was first designed by Frank et al., in 1993 with the use of acoustic foil inside it [1]. Later, this ultrasonic concentrator design was improved by Hill

\footnotetext{
* Corresponding author.

E-mail address: khinnwezintun@gmail.com
}

https://doi.org/10.37934/arfmts.79.2.7482 
and Wood in which the use of acoustic foil was omitted. The Martyn Hill [2] designed the layer of resonators for ultrasonic particle manipulation by using one dimensional simulation model. This study observed different coupling layer materials (brass, aluminium, macor) of quarter wavelength resonator predicted the nodal position and device behaviour. Süleyman Büyükkoçak et al., [3] discussed the particle trajectories of $Y$-shaped channel. Fluid flow inside the microchannel geometry operated at resonance frequency was modeled FEM based numerical simulation. The numerical prediction was validated with experimental result from the literature. In a similar study the locations of particles were predicted using a finite element model by Adrian Neild et al., [4]. They used simple model with resonance frequency to estimate the number of lines formed at this channel. Henrik Bruus [5] described the theory of acoustic radiation force on a second order, time-average effect, micrometer sized particles in ultrasonic field. Microchip-based devices for on-line particle separation were developed by Andreas Nilsson [6]. This on-line separation, $90 \%$ of the particles were recovered by using the acoustic separator at fundamental resonance frequency $2 \mathrm{MHz}$. Most of the models discussed earlier are based on one-dimensional analysis. The two-dimensional modelling of the separation of suspended particle as a function of time was reported by Francisco J. Trujillo et al., in 2013 by using finite element analysis [7].

Most studies focused on separation, trapping or concentration of microparticles. This study aims to concentrate nanoparticles using the ultrasonic standing wave field and gravity-aided sedimentation. Separation performance of macro-scale acoustic concentrator in flow field was investigated by experimental tests and two-dimensional acoustic simulation model was also constructed to qualitatively analyze variation of sound field inside the fluidic channel.

\section{Theory}

The acoustic radiation force was calculated using Gor'kov's formulation [8,15]. The acoustic radiation force is defined as a function of a potential $U$.

$F_{a}=-\nabla U$

The force is the negative gradient of the force potential [10]. Where the potential $U$ is defined as:

$$
\begin{aligned}
& \mathrm{U}=2 \pi \rho_{\mathrm{w}} \mathrm{r}^{3}\left[\frac{\mathrm{f}_{1}}{3 \rho_{\mathrm{w}}{ }^{2} \mathrm{c}_{\mathrm{w}}{ }^{2}}\langle p\rangle^{2}-\frac{\mathrm{f}_{2}}{2}\langle\mathrm{v}\rangle^{2}\right] \\
& \mathrm{f}_{1}=1-\frac{\rho_{\mathrm{w}} \mathrm{c}_{\mathrm{w}}{ }^{2}}{\rho_{\mathrm{p}} \mathrm{c}_{\mathrm{p}}{ }^{2}} \quad, \quad \mathrm{f}_{2}=\frac{2\left(\rho_{\mathrm{p}}-\rho_{\mathrm{w}}\right)}{2 \rho_{\mathrm{p}}+\rho_{\mathrm{w}}}
\end{aligned}
$$

where, $r$ is the radius of particles, $c$ is the speed of sound, $\rho$ is the density, $p$ is the acoustic pressure and $v$ is the fluid particles velocity, the subscripts $w$ and $p$ refer to water and particle properties. The wave number $k$ is defined by $2 \pi / \lambda$.

The particle movement either to the pressure node or anti-node is determined by the sign of acoustic contrast factor $\varnothing$ which depends on the densities and compressibility of fluid and particles [11].

$\varnothing=\frac{1}{3}\left(\frac{5 \rho_{p}-2 \rho_{w}}{2 \rho_{p}+\rho_{w}}-\frac{\rho_{w} c_{w}^{2}}{\rho_{p} c_{p}^{2}}\right)$ 
The prominent forces acting on the particle under ultrasonic standing wave and flow fields are primary acoustic radiation force, lateral radiation force, Stokes drag forces [12]. The stokes drag force $F_{\text {drag }}$ can be expressed as

$$
F_{\text {drag }}=6 \pi \mu r\left(v_{f}-v_{p}\right)
$$

For a spherical particle with radius $r$, moving at the fluid and particle velocity are $v_{f}$ and $v_{p}$ through a liquid with viscosity $\mu$. The effect of secondary radiation force and interparticle forces are not considered in the simulation model.

The relative densities of the particle and fluid control, the buoyancy force on a particle, where $F_{B}$ is opposite to the gravitational force [13]

$$
F_{b}=\frac{4}{3} \pi r^{3} g\left(p_{w}-p_{p}\right)
$$

where $r$ is the radius of the particle, $g$ is the gravitational acceleration, $\rho_{w}$ and $\rho_{p}$ are the densities of the fluid and the particle. When referenced to the $x$ and $y$ coordinates of the particle, the $x$ and $y$ components of the buoyancy force depend on the orientation of the channel.

\section{Experimental Setup}

The experimental setup involves four main parts, a function generator, an acoustic chamber, syringe pump and an oscilloscope as shown in Figure 1. The function generator (TEXIO, FGX-2005) is a power source to the piezoelectric transducer. The purpose of using a piezoelectric transducer is to transmit waves in the ultrasonic frequency range to the aluminum channel. This creates acoustic standing waves on the channel that will result in acoustic radiation force on the particles. Oscilloscope (GDS-1102A-U) is used to monitor the amplitude and frequency of the input voltage to the separator. Syringe pump (FGX-2005) is used to introduce the water-particle solution into the acoustic separator through the connector at desired flow rate.

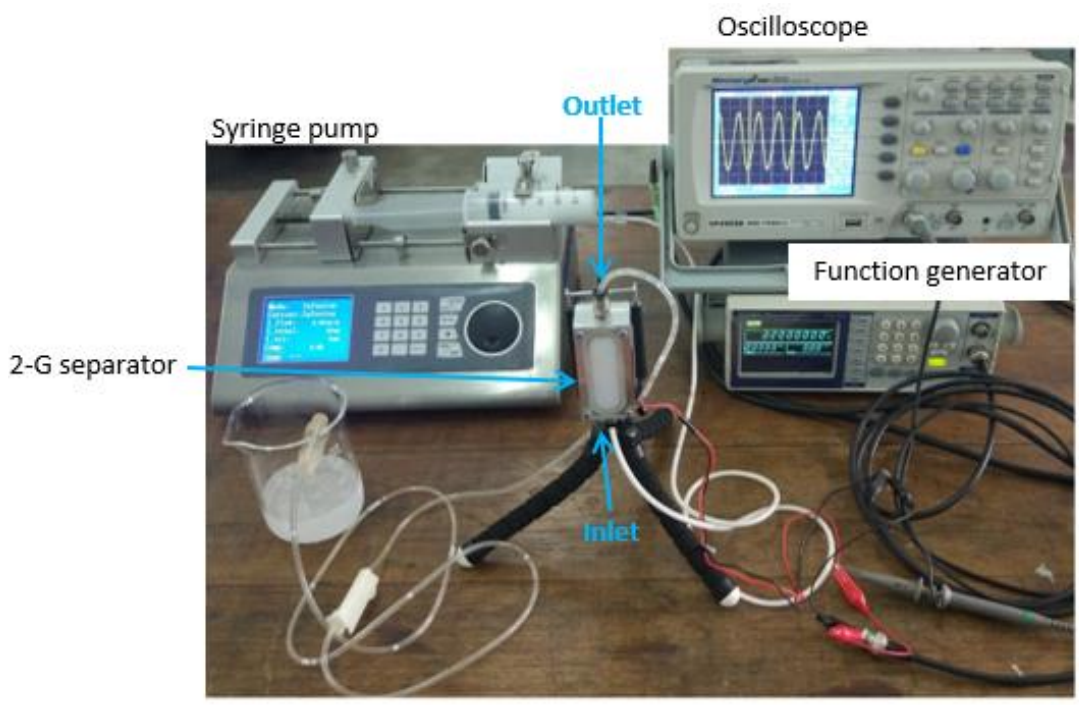

Fig. 1. Experiment Setup 
The operating principle of the device is shown in Figure 2. Initially, the fluid-particle solution is introduced into the channel by the syringe pump. When ultrasonic wave is generated by the piezoelectric transducer excited by the function generator, the particles experience the acoustic radiation forces and the Stokes drag force which are induced by the ultrasonic standing wave and flow fields [9]. If the primary radiation force is larger than drag force and they will move to the pressure nodes [14]. The separation lines are more prominent at the resonant frequency of the fluid layer. Then, particles will form agglomerates due to secondary and interparticle forces. When agglomerates are large enough, gravitational force can overcome the Stokes drag force and the trapped particles will settle down when they reach to critical volume. Therefore, the clean fluid is flowed through the outlet and the settled particles will remain in the separator near inlet of the fluid flow. One of the drawbacks of this design is the blockage of particles concentrated at the bottom of the channel near the inlet resulting in intermittent operation of the device.

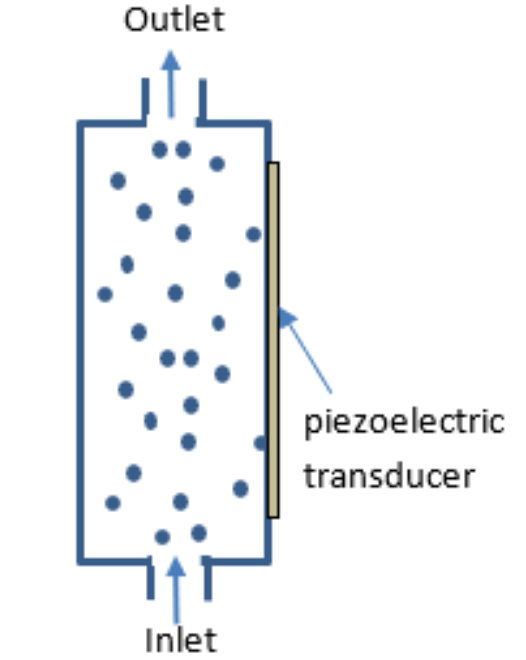

(fluid with suspended particles)

(a)

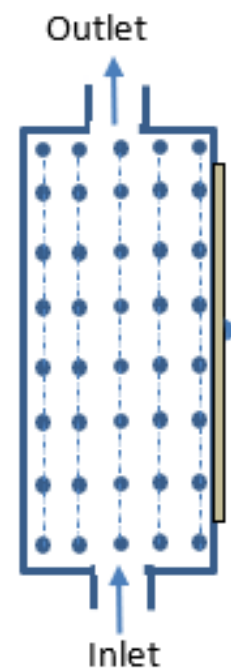

(fluid with suspended particles)

(b)

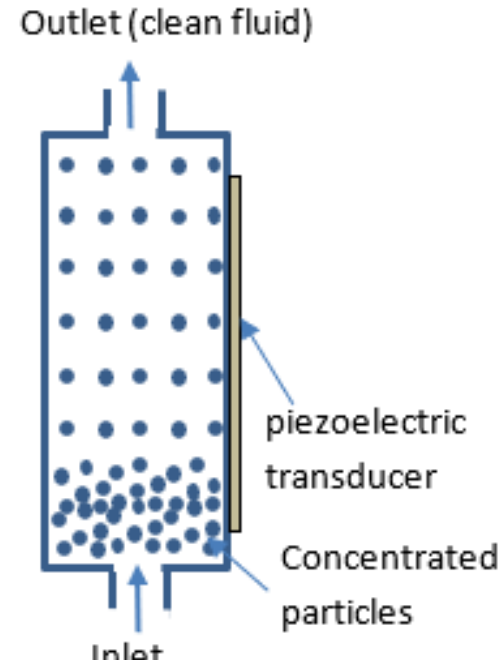

(fluid with suspended particles)

(c)

Fig. 2. Demonstration of device operation (a) Ultrasound is off and particles spread across the channel with the flow, (b) Ultrasound is on and particles form lines at pressure nodes under the primary radiation force, (c) Particles form agglomerate due to secondary radiation force and fall down due to gravity

\section{Numerical Analysis}

This simulation was performed using commercial finite element package, COMSOL Multiphysics software in pressure acoustic module in frequency domain. The channel is treated as two dimensional model of fluid layer. The domain is the rectangle with $0.016 \mathrm{~m}$ width and $0.04 \mathrm{~m}$ length. For this simulation, maximum mesh size of the element is around $\lambda / 10$ so that it is sufficient to capture the variation of acoustic parameters inside the channel. The input parameters for the simulations are density of water $\rho_{w}=1000 \mathrm{~kg} / \mathrm{m}^{3}$, viscosity $\mu=8.94 \times 10^{-4} \mathrm{~kg} /(\mathrm{m} \bullet \mathrm{s})$, speed of sound of water $c=1500 \mathrm{~m} / \mathrm{s}$, particle diameter $=40 \mathrm{~nm}$ and density of particle $\rho_{p}=3780 \mathrm{~kg} / \mathrm{m}^{3}$.

Figure 3 shows the simplified model with boundary conditions. All the rigid walls of the channel were modelled as sound hard boundaries, except the wall where the piezo-actuator was attached. At this side of the model was simplified by using normal acceleration boundary. 


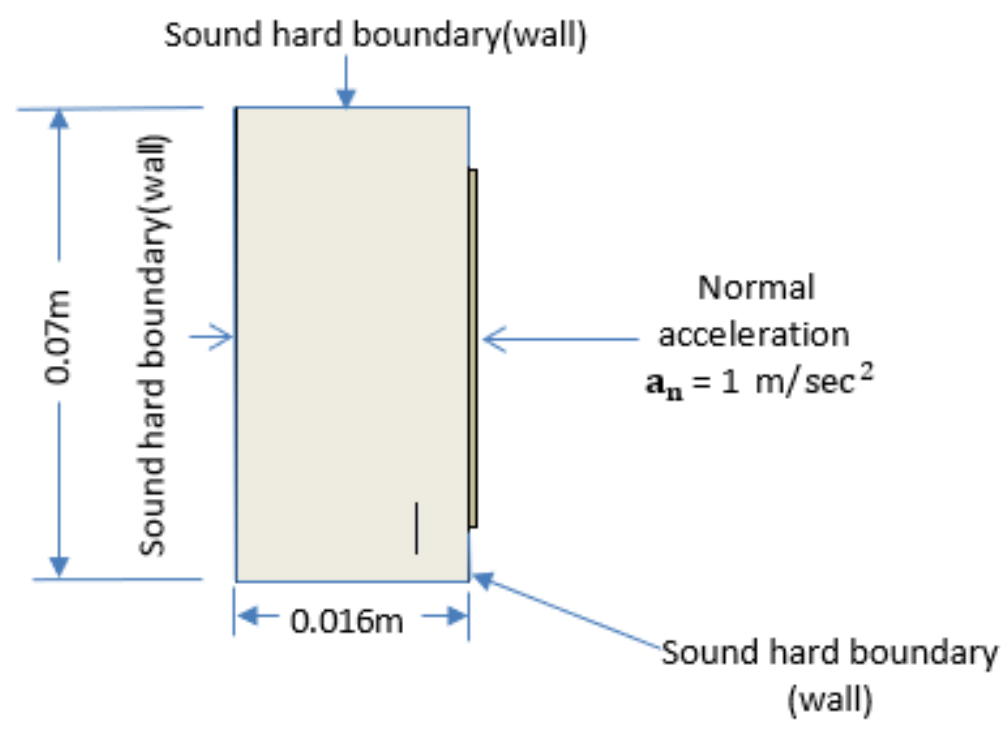

Fig. 3. Two-dimensional channel geometry

$-n \cdot\left(-\frac{1}{\rho}\left(\nabla p_{t}-q_{d}\right)\right)=a_{n}$

The sound hard boundary is defined by

$-n \cdot\left(-\frac{1}{\rho}\left(\nabla p_{t}-q_{d}\right)\right)=0$

Acoustic radiation force is calculated by

$F_{a c}=\frac{1}{3 \rho_{f} \omega^{2}} \pi r^{3} k \varphi\left[\frac{\partial \hat{P}(x, y)}{\partial x}\left(\frac{\partial \hat{P}(x, y)}{\partial x}\right)^{*}+\frac{\partial \hat{P}(x, y)}{\partial y}\left(\frac{\partial \hat{P}(x, y)}{\partial y}\right)^{*}+\frac{\omega^{2}}{c_{f}^{2}} \hat{P} \hat{P}^{*}\right] \sin (2 k x)$

where, $p_{t}=$ Total acoustic pressure field

$a_{\mathrm{n}}=$ normal acceleration

$\mathrm{q}_{\mathrm{d}}=$ Dipole domain source

$r=$ radius of particle

\section{Results and Discussion}

\subsection{Experimental Result}

The fluid particle solution is introduced into the separator by the syringe pump through the inlet at the flow rate of $0.1 \mathrm{ml} / \mathrm{min}$. The particles experience the acoustic radiation force which is provided by the ultrasonic standing wave field and will move to the pressure node points. The separation lines or node points are prominent at the resonance frequency of $2.2 \mathrm{MHz}$ as shown in Figure 4. 


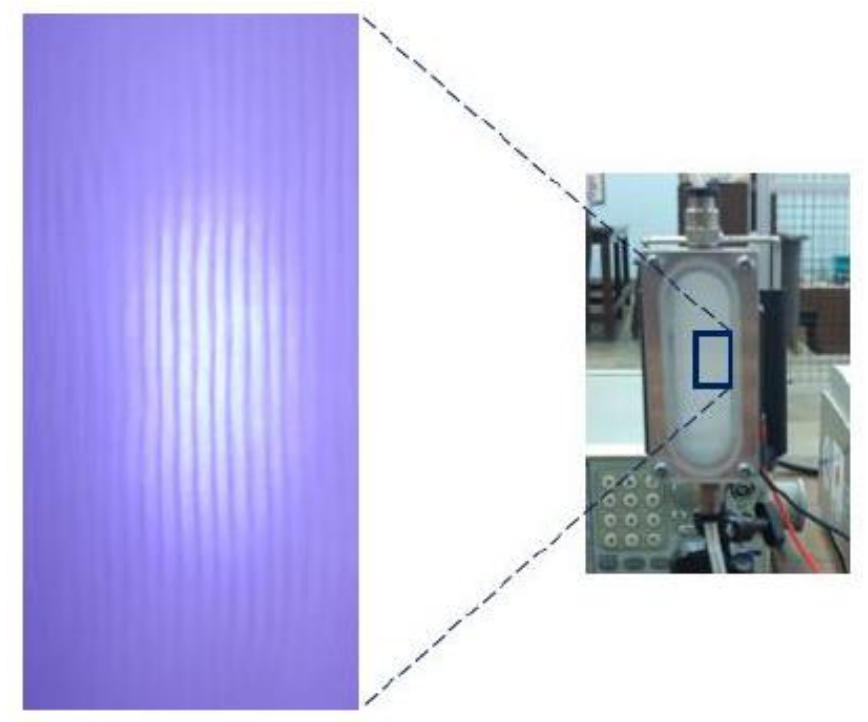

Fig. 4. Pressure nodal lines in the fluid layer of the ultrasonic separator under the influence of primary radiation force

The particles in the nodal lines then form aggregates under the influence of the later radiation force and secondary interparticle forces. The aggregates of particles settle down when they reach to critical volume. Therefore, the clean fluid is flowed through the outlet into the beaker and the settled particles will remain in the separator near inlet of the fluid flow. At the end of the concentration experiment when particle sedimentation starts to disturb the inlet flow, the operation is stopped and fluid samples from inlet solution and outlet solution are put on the glass slide under the microscope Keyence VHX-5000. The distribution of particles in the samples from the inlet and outlet of the separator are shown in Figure 5. As can be seen in Figure 5, particle concentration is higher in the inlet and lower in the outlet. Separation performance of the device is computed by measuring the areas occupied by the particle aggregates in the samples from inlet and outlet. The separation efficiency of the device is found to be $82.85 \%$ and the particles are concentrated at the bottom of the channel.

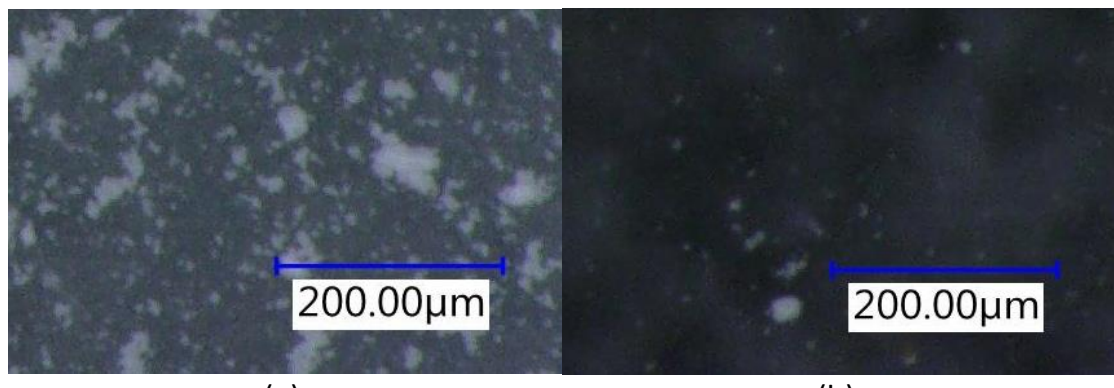

(a)

(b)

Fig. 5. Particle distribution in the sample fluid (a) Sample from the inlet (b) Sample from the outlet 


\subsection{Numerical Result}

To understand the variation of sound field inside the channel, acoustic simulation was conducted in COMSOL Multiphysics. Figure 6(a) shows two-dimensional numerical simulation results for acoustic pressure distribution in the separator. The equally spaced lines along the separator show that standing wave is constructed across the separator width. According to Figure $6(\mathrm{a})$, the maximum acoustic pressure is $4 \times 10^{4} \mathrm{~Pa}$ and the minimum acoustic pressure is $-4 \times 10^{4} \mathrm{~Pa}$. Absolute displacement velocity in the separator is plotted in Figure $6(\mathrm{~b})$. From the comparison, it can be clearly seen that the velocity and the pressure plots have phase difference since the theoretical phase difference between the pressure and velocity is $\pi / 2$.

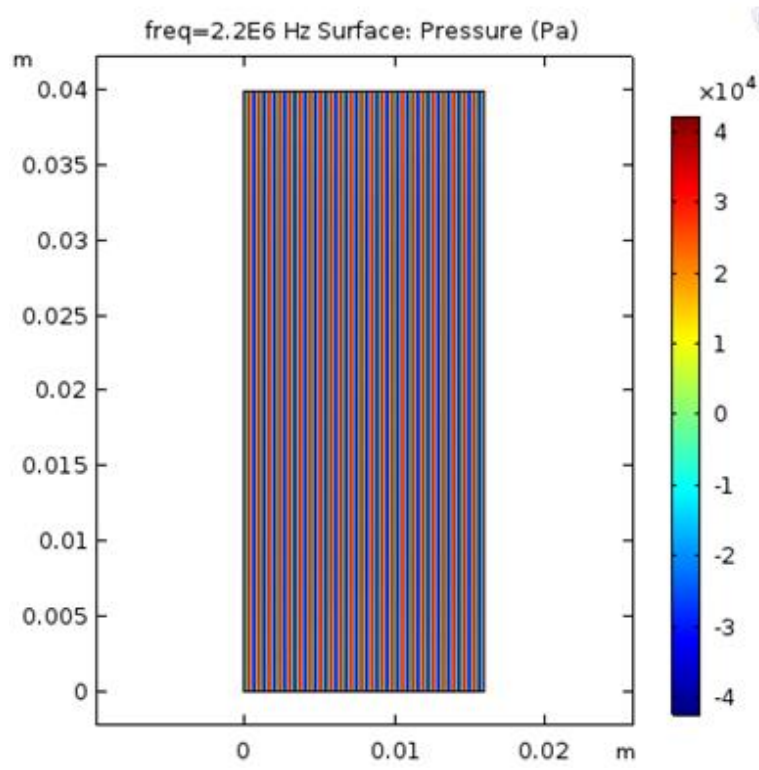

(a)

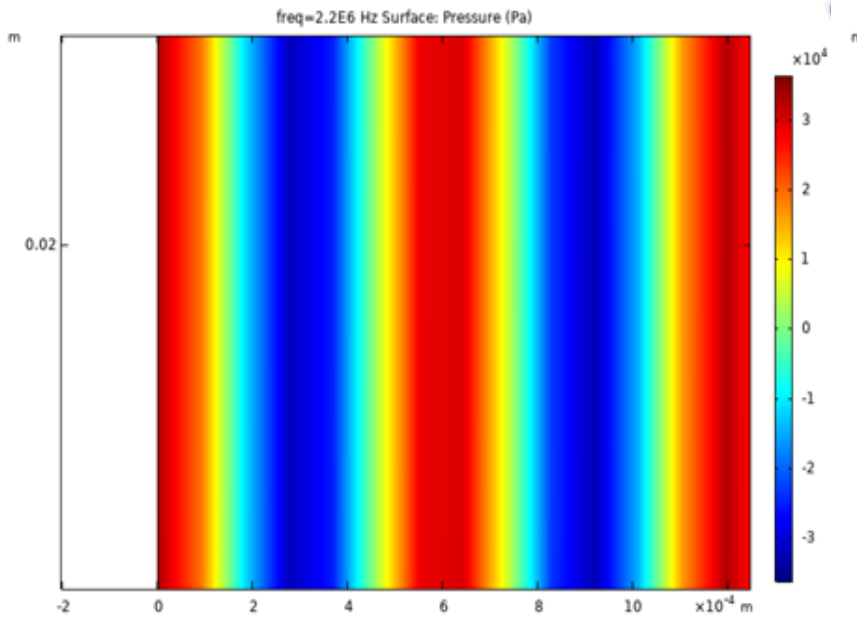

(c)

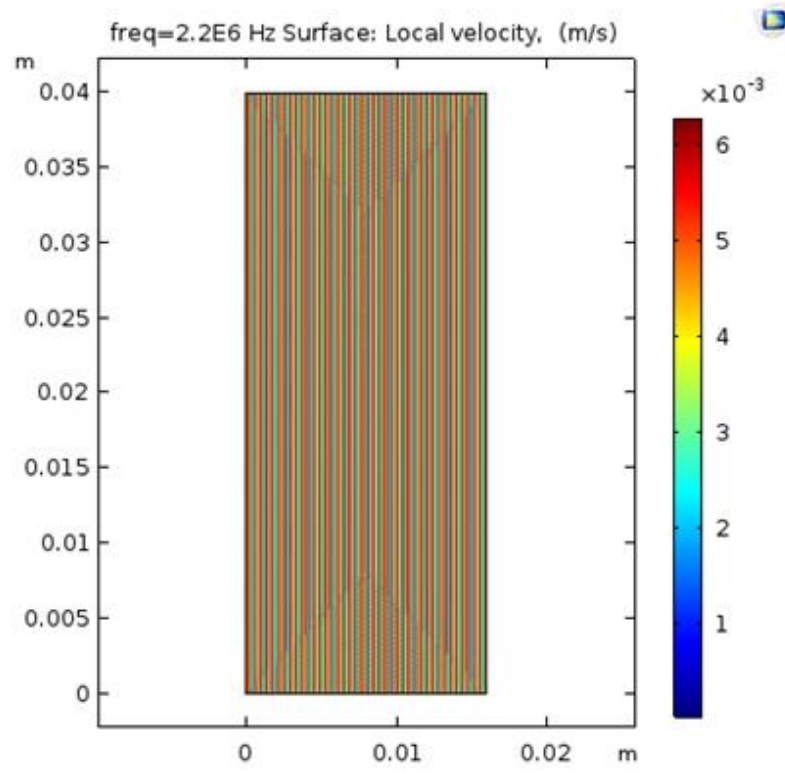

(b)

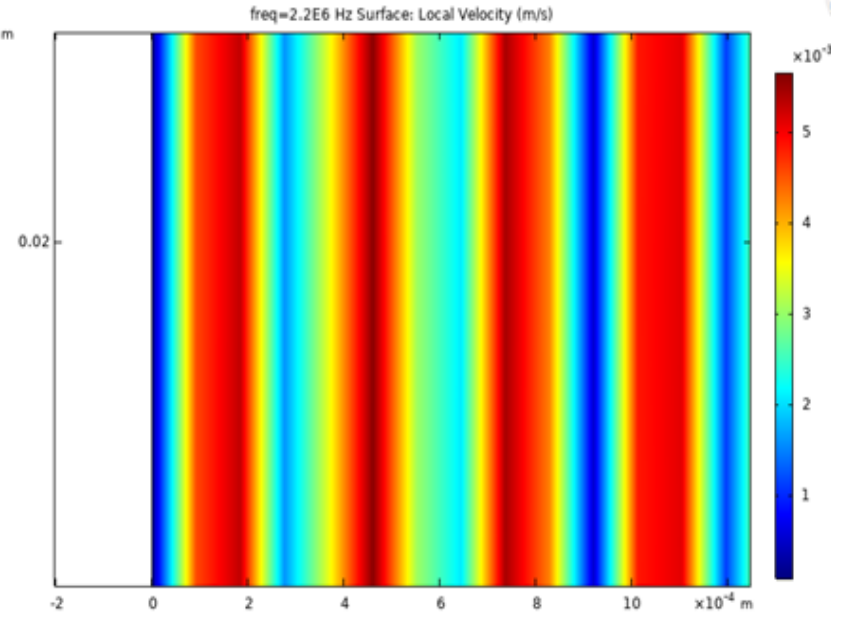

(d)

Fig. 6. Two-dimension model of the separator (a)Acoustic Pressure and (b) Displacement Velocity Distribution (c) Acoustic Pressure near the rigid boundary (d) Displacement velocity near the rigid boundary

Primary acoustic radiation force acting on the particles was computed using Eq. (9) assuming primary radiation force acts on the individual particle. Variation of acoustic radiation force is shown in Figure 7. From Figure 7, It can be seen that the maximum acoustic radiation force along the $x$ direction is $2 \times 10^{-11} \mathrm{~N}$, which is in the same order of magnitude for the primary radiation force [8]. 
Moreover, it can be clearly seen that the lateral radiation force in the y-directions as shown by the variation of the radiation in the y-direction in Figure 7. This lateral radiation force is responsible for the forming of particle aggregates after they form the lines at the pressure nodal lines. It must be noted here that this two dimensional model under unit normal acceleration excitation can only characterize the sound field variation in the channel qualitatively and therefore absolute magnitude of the radiation forces cannot be obtained from the simulation model.

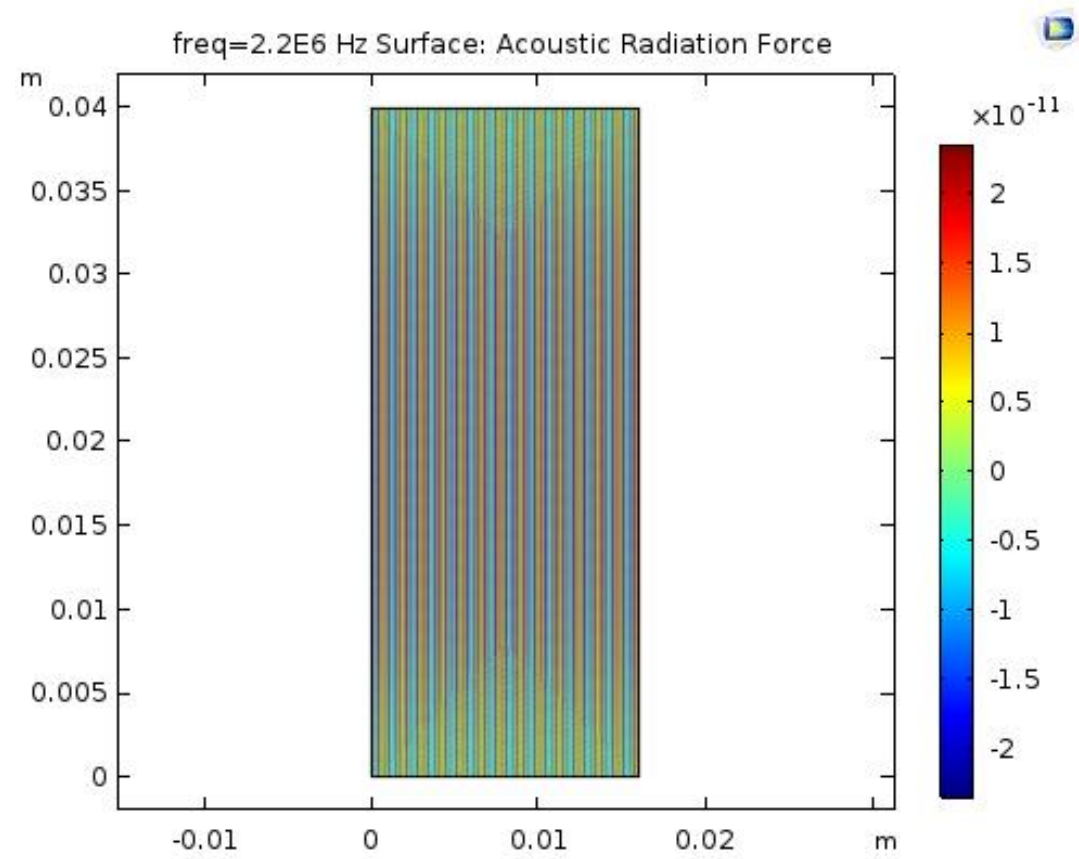

Fig. 7. Two-dimension model for acoustic radiation force

\section{Conclusions}

In this paper, the separation of nanoparticles from suspending fluid was done using ultrasonic standing wave technology and gravity-aided sedimentation. Titanium dioxide nanoparticles were found to respond to primary acoustic radiation force at operating frequency $2.2 \mathrm{MHz}$ and they formed agglomerates very quickly due to lateral radiation force as well as secondary radiation force. Once the agglomerates become large enough, they sediment at the bottom of the channel due to gravity. In this way, separation effect was achieved. The experimental results show that separation efficiency of $82.85 \%$ can be achieved at a flow rate is $0.1 \mathrm{ml} / \mathrm{min}$. However, the flow rate is still very low and the optimization of channel design is necessary for enhancing the flow rate. Other modes of operation such as sweeping mode could also assist to enhance flow rate. Future research should focus on optimization of the ultrasonic separation design with other modes of operation. Blockage of the channel after particles sediment near the inlet of the channel should also be addressed in the future study. Simulation results indicated that there is lateral radiation force in the resonator which is responsible for formation particle agglomerates hence in good agreement with experimental observation. Moreover, simulation model could be extended to 3D to investigate three-dimensional variation of the sound field inside the channel. Simulation of coupled flow and acoustic field with particle trajectory model should also be done to identify separator's performance and it could be used for multiphysics optimization of the performance of the channel. 


\section{References}

[1] FRANK, F. "Separartion of suspended particles by use of the inclined resonator concept." In Ultrasonics Int. 93 Conf. Proc. 1993.

https://doi.org/10.1016/B978-0-7506-1877-9.50129-3

[2] Hill, Martyn, Rosemary J. Townsend, and Nicholas R. Harris. "Modelling for the robust design of layered resonators for ultrasonic particle manipulation." Ultrasonics 48, no. 6-7 (2008): 521-528.

https://doi.org/10.1016/j.ultras.2008.06.007

[3] Büyükkoçak, Süleyman, Barbaros Çetin, and Mehmet Bülent Özer. "Finite Element Modeling of Micro-Particle Separation Using Ultrasonic Standing Waves." In International Conference on Nanochannels, Microchannels, and Minichannels, vol. 46278, p. V001T12A005. American Society of Mechanical Engineers, 2014. https://doi.org/10.1115/ICNMM2014-21436

[4] Neild, Adrian, Stefano Oberti, Albrecht Haake, and Jürg Dual. "Finite element modeling of a microparticle manipulator." Ultrasonics 44 (2006): e455-e460.

https://doi.org/10.1016/i.ultras.2006.05.168

[5] Bruus, Henrik. "Acoustofluidics 7: The acoustic radiation force on small particles." Lab on a Chip 12, no. 6 (2012): 1014-1021. https://doi.org/10.1039/c2lc21068a

[6] Nilsson, Andreas, Filip Petersson, Henrik Jönsson, and Thomas Laurell. "Acoustic control of suspended particles in micro fluidic chips." Lab on a Chip, no. 2 (2004): 131-135.

https://doi.org/10.1039/B313493H

[7] Trujillo, Francisco J., Sebastian Eberhardt, Dirk Möller, Jurg Dual, and Kai Knoerzer. "Multiphysics modelling of the separation of suspended particles via frequency ramping of ultrasonic standing waves." Ultrasonics sonochemistry 20, no. 2 (2013): 655-666. https://doi.org/10.1016/j.ultsonch.2012.08.014

[8] Courtney, Charles RP, C-K. Ong, B. W. Drinkwater, A. L. Bernassau, P. D. Wilcox, and D. R. S. Cumming. "Manipulation of particles in two dimensions using phase controllable ultrasonic standing waves." Proceedings of the Royal Society A: Mathematical, Physical and Engineering Sciences 468, no. 2138 (2012): 337-360. https://doi.org/10.1098/rspa.2011.0269

[9] Hill, Martyn, Yijun Shen, and Jeremy J. Hawkes. "Modelling of layered resonators for ultrasonic separation." Ultrasonics 40, no. 1-8 (2002): 385-392. https://doi.org/10.1016/S0041-624X(02)00127-0

[10] Dual, Jürg, Philipp Hahn, Ivo Leibacher, Dirk Möller, Thomas Schwarz, and Jingtao Wang. "Acoustofluidics 19: Ultrasonic microrobotics in cavities: devices and numerical simulation." Lab on a Chip 12, no. 20 (2012): 4010-4021. https://doi.org/10.1039/c2lc40733g

[11] Cetin, Barbaros, Mehmet Bülent Özer, and Mehmet Ertuğrul Solmaz. "Microfluidic bio-particle manipulation for biotechnology." Biochemical engineering journal 92 (2014): 63-82. https://doi.org/10.1016/i.bej.2014.07.013

[12] Liu, Yang, and Kian-Meng Lim. "Particle separation in microfluidics using a switching ultrasonic field." Lab on a Chip 11, no. 18 (2011): 3167-3173. https://doi.org/10.1039/c1lc20481e

[13] Shin, Beom-Soo, and M. C. Danao. "Characteristics of particle separation in suspension using an ultrasonic standing wave." J. Biosyst. Eng 37, no. 2 (2012): 113-121. https://doi.org/10.5307/JBE.2012.37.2.113

[14] Guldiken, Rasim, Myeong Chan Jo, Nathan D. Gallant, Utkan Demirci, and Jiang Zhe. "Sheathless size-based acoustic particle separation." Sensors 12, no. 1 (2012): 905-922. https://doi.org/10.3390/s120100905

[15] Rogers, Priscilla, Ian Gralinski, Cameron Galtry, and Adrian Neild. "Selective particle and cell clustering at air-liquid interfaces within ultrasonic microfluidic systems." Microfluidics and nanofluidics 14, no. 3-4 (2013): 469-477.

https://doi.org/10.1007/s10404-012-1065-9 